\title{
New Ways to Look at Old Patterns? Sequence Analysis to Analyze Patterns of Clinical Stability in Community-Acquired Pneumonia
}

\author{
Marisha Burden, $M D^{7}$ and Eric M. Mortensen, MD, MSc ${ }^{2}$ \\ 'Denver Health, Denver Colorado and University of Colorado School of Medicine, Aurora, CO, USA; ${ }^{2}$ VA North Texas Health Care System and \\ University of Texas Southwestern Medical Center, Dallas, TX, USA.
}

J Gen Intern Med 29(4):554-5

DOI: $10.1007 / \mathrm{s} 11606-013-2760-2$

(c) Society of General Internal Medicine 2014

\begin{abstract}
A nnually, over one million patients are hospitalized for community-acquired pneumonia in the United States, accounting for greater than 2 billion dollars in health care spending, and with a short-term mortality rate of up to $36 \% .{ }^{1,2}$ Despite extensive research and clinical practice guidelines that provide evidence-based guidance regarding the diagnosis and management of community-acquired pneumonia, it continues to be the leading cause of infectious death nationwide. ${ }^{3}$ Studies have shown that there are substantial variations in care delivered to patients with pneumonia that are not due to the severity of disease alone. ${ }^{1,4}$ A major part of the complexity of caring for these patients is that health care providers need to determine whether to admit a patient, to what type of care setting (i.e., observation unit, floor unit, or intensive care unit), and to determine when the patient can be safely discharged. Both the Pneumonia Severity Index (PSI) and CURB-65 are scoring systems that aid in the admission decision, but both are limited by being based upon data at the time of admission when in reality patient clinical trajectories are dynamically evolving. ${ }^{2}$ This results in approximately $45 \%$ of patients with community-acquired pneumonia who ultimately require intensive care unit admission initially being admitted to a non-intensive care unit setting. ${ }^{5}$ A similar problem presents itself at discharge. Previous research has shown that indicators of clinical stability in pneumonia (such as fever, heart rate, respiratory rate, systolic blood pressure, ability to maintain oral intake, and mentation) can be utilized to help determine when a patient is ready for discharge, ${ }^{6}$ but whether any, and if so, which patients can be discharged with clinical instabilities is debated. Accordingly, there are still many opportunities for improving the care of patients hospitalized with community-acquired pneumonia.
\end{abstract}

Published online January 16, 2014
In the 1960 s, scientists began using sequencing methodology to identify the relatedness of homogeneous groups of amino acid sequences in large, heterogeneous data sets to determine how related proteins may have evolved. $^{7}$ A similar methodology has been utilized in other disciplines, including the social sciences (e.g., studying career trajectories), archaeology (e.g., tree ring dating of rooms in pueblos), economics (e.g., trends in markets), and linguistics (e.g., how children learn a language). ${ }^{8}$ The methodology of sequence analysis may seem initially daunting, but may also have important implications for how future studies are conducted in medicine. Sequences of events can be treated as whole units and the units can then be analyzed for discrete patterns of interest. Simply put, sequence analysis sets the stage to not just analyze data in units or attributes, but also to investigate temporality, that is, patients' clinical trajectories over time. ${ }^{8}$

In this month's Journal, Hougham et al. present a novel take on sequence analysis in a study of patients hospitalized with community-acquired pneumonia. ${ }^{9}$ Their study examines whether the temporal order in which clinical indicators resolve, including fever, tachycardia, tachypnea, hypotension, hypoxia, inability to maintain oral intake, and altered mental status, is associated with 30-day mortality, length of hospital stay, and total costs. They were able to identify eight major unique clusters of sequences by which the clinical indicators resolved (or instabilities remained at discharge) in 1,326 adult patients hospitalized with community-acquired pneumonia. Patients in each of these "clusters" had significant differences in both health and resource utilization outcomes. For example, patients in the Febrile, Tachycardic, and Tachycardic/Hypoxic clusters, which still had at least one instability unresolved at discharge, had 30-day mortality rates that were similar to patients in the two Stable clusters for which all clinical instabilities were resolved by discharge. In contrast, patients in the Mental/Oral/Hypoxic group had an markedly higher odds of death within 30 days (odds ratio 3.99, $95 \%$ CI $1.68-9.50)$.

In 1998, Halm et al. demonstrated that the median time to stability of vital signs for patients with community-acquired 
pneumonia was 3 days using the most lenient definition of stability, and 7 days using the most conservative definition, and subsequently reported that the likelihood of death or readmission was related to whether patients were discharged without resolution of one or more of the abnormal vital signs. ${ }^{6,10}$ Hougham et al. add to this literature by suggesting that the timing and order of resolution of vital signs may also be predictive for important clinical outcomes such as mortality and length of stay.

Sequence analysis in clinical medicine is rather new, and additional studies will be needed to assess its utility. The methodology is complex, and Hougham and colleagues were not able to account for those who had vital signs that might have de-stabilized prior to discharge, which the authors point out would be even more complex to study. In addition, a number of the clusters identified had boundaries that were near the boundaries of other clusters. Finally, there were more than 900 stability patterns for the 1,386 patients in their study, which demonstrates both the limitations of this methodology and the heterogeneity in how these instabilities resolve for patients hospitalized with community-acquired pneumonia.

In closing, Hougham et al. have used a novel methodology to study the patterns of resolving clinical instabilities in community-acquired pneumonia. This methodology may aid future studies of not only pneumonia, but also other conditions such as heart failure, in addition to a broad array of clinical phenomenon such as readmissions and health care utilization.

Acknowledgements: This material is the result of work supported with resources and facilities of the VA North Texas Health Care System. The views expressed in this article are those of the author and do not necessarily represent the views of the Department of Veterans Affairs.

Corresponding Author: Eric M. Mortensen, MD, MSc; Dallas VA Medical Center, General Internal Medicine (111E), 4500 South Lancaster, Dallas, TX 75216, USA (e-mail: Eric.mortensen@ utsouthwestern.edu).

\section{REFERENCES}

1. Fine MJ, Medsger AR, Stone RA, et al. The hospital discharge decision for patients with community-acquired pneumonia. Results from the pneumonia patient outcomes research team cohort study. Arch Intern Med. 1997; 157:47-56.

2. Niederman MS, Mandell LA, Anzueto A, et al. Guidelines for the management of adults with community-acquired pneumonia. Diagnosis, assessment of severity, antimicrobial therapy, and prevention. Am J Respir Crit Care Med. 2001;163:1730-54.

3. Murphy SXJ, and Kochanek K. Deaths: Final Data for 2010. National Vital Statistics Reports 2013;61.

4. Fine MJ, Singer DE, Phelps AL, Hanusa BH, Kapoor WN. Differences in length of hospital stay in patients with community-acquired pneumonia: a prospective four-hospital study. Med Care. 1993;31:371-80.

5. Mandell LA, Wunderink RG, Anzueto A, et al. Infectious Diseases Society of America/American Thoracic Society consensus guidelines on the management of community-acquired pneumonia in adults. Clin Infect Dis. 2007;44(Suppl 2):S27-72.

6. Halm EA, Fine MJ, Marrie TJ, et al. Time to clinical stability in patients hospitalized with community-acquired pneumonia: implications for practice guidelines. JAMA. 1998;279:1452-7.

7. Needleman SB, Wunsch CD. A general method applicable to the search for similarities in the amino acid sequence of two proteins. J Mol Bio. 1970;48:443-53

8. Abbott A. Sequence analysis: New Methods for Old Ideas. Annu Rev Sociol. 1995;21:93-113.

9. Hougham GW, Ham SA, Ruhnke GW, et al. Sequence Patterns in the Resolution of Clinical Instabilities in Community-Acquired Pneumonia and Association with Outcomes. Journal of general internal medicine DOI: $10.1007 / \mathrm{s} 11606-013-2626-7$.

10. Halm EA, Fine MJ, Kapoor WN, Singer DE, Marrie TJ, Siu AL. Instability on hospital discharge and the risk of adverse outcomes in patients with pneumonia. Arch Intern Med. 2002;162:1278-84. 